\title{
Effects of Quality Control Circle Activities for Intravenous Cannula Placement Using Deming Cycle Management: A Case-Controlled Study
}

\author{
Yahui Zhao", Huiling Liu ${ }^{1,}$,, Qingqing Zhai ${ }^{1}$, Mengying $\mathrm{Qi}^{2}$, Xiaolan Wang ${ }^{3}$, Yanhua $\mathrm{Shi}^{3}$ \\ ${ }^{1}$ Department of External Renal Thoracic Surgery, The First Affiliated Hospital of Jinan University, Guangzhou, China \\ ${ }^{2}$ School of Nursing, Jinan University, Guangzhou, China \\ ${ }^{3}$ Department of Hepatobiliary Surgery, The First Affiliated Hospital of Jinan University, Guangzhou, China \\ Email address: \\ huilinghappy@163.com (Yahui Zhao),2827758463@qq.com (Huiling Liu), wangxiaolan74@126.com (Xiaolan Wang), \\ 347039102@qq.com (Mengying Qi), 1187113986@qq.com (Qingqing Zhai), 59235310@qq.com (Yanhua Shi) \\ ${ }^{*}$ Corresponding author
}

\section{To cite this article:}

Yahui Zhao, Huiling Liu, Qingqing Zhai, Mengying Qi, Xiaolan Wang, Yanhua Shi. Effects of Quality Control Circle Activities for Intravenous Cannula Placement Using Deming Cycle Management: A Case-Controlled Study. American Journal of Nursing Science.

Vol. 10, No. 1, 2021, pp. 59-63. doi: 10.11648/j.ajns.20211001.21

Received: November 3, 2020; Accepted: February 9, 2021; Published: February 23, 2021

\begin{abstract}
The present study aimed to explore the effectiveness of quality control circle (QCC) activities under the guidance of the Deming cycle Management for improving intravenous (IV) cannula placement. A total of 1,035 patients who underwent IV cannula placement from March 2019 to June 2019 in the Department of Hepatobiliary Surgery and Department of Renal and Thoracic Surgery of our hospital were enrolled as the control group, and 1,437 patients who underwent IV cannula placement in the same places as above but from July 2019 to December 2019 were enrolled as the test group. A between-group comparison was performed on intangible and tangible outcomes (duration of IV cannula placement, complications, patient awareness rate of maintenance knowledge of IV cannula, patient satisfaction rate with nursing staff, nurse skills in IV cannula infusion, and maintenance). After QCC activities were implemented, the scores of 11 QCC members in the five aspects of QCC technique application, communication and coordination, sense of responsibility and honor, motivation, and problem-solving ability all improved compared with those before the activities $(\mathrm{p}<$ 0.05 ), and the duration of IV cannula placement in the test group was $70.22 \pm 48.33 \mathrm{~h}$, which was longer $(\mathrm{p}<0.05)$ than the $60.59 \pm 41.9$ $\mathrm{h}$ of the control group. The test group had a significantly lower $(\mathrm{p}<0.05)$ rate of complications $(12.9 \%, 12.6 \%$, and $1.3 \%$ for extravasation, phlebitis, and cannula obstruction, respectively) than the control group $(30.0 \%, 11.4 \%$, and $4.1 \%$ for blood oozing, phlebitis, and cannula obstruction respectively); the patient awareness rate of maintenance knowledge of IV cannula and patient satisfaction rate with nursing staff in the test group were $90 \%$ and $96.3 \%$, respectively, which were significantly higher $(\mathrm{p}<0.05)$ than the $82.4 \%$ and $81.5 \%$ in the control group. The scores of nurse skills in IV cannula infusion and maintenance in the test group were $90.5 \pm 1.3$ and $93.6 \pm 2.0$, respectively, which were significantly higher $(\mathrm{p}<0.05)$ than the $85.5 \pm 2.7$ and $81 \pm 1.0$ in the control group. The implementation of QCC activities under the guidance of the Deming cycle Management in IV cannula placement improves the ability of the department's nursing team to identify, analyze, and solve problems, which is favorable for continuous improvement of nursing quality, leading to an elongated duration of IV cannula placement, reduced rate of complications, and improved patient satisfaction rate with nursing services.
\end{abstract}

Keywords: Intravenous Cannula Placement, Deming Cycle, Quality Control Circle, Placemat Duration, Complications

\section{Introduction}

The Plan-Do-Check-Act (PDCA) cycle was proposed by the American quality management expert Deming in the 1950 s and is also known as the Deming cycle [1]. It refers to the cyclic process in all management activities to improve management quality and efficiency. This scientific management method is now widely used in health care management [2]. Peripheral intravenous (IV) catheters (PIVCs) have gained popularity in Europe and the United 
States since the 1960s, with approximately 2 billion PIVCs consumed annually across the globe [3]. IV cannulas are one of the most common types of tools used for peripheral intravenous infusion, and studies have shown that more than $70 \%$ of hospitalized patients use IV cannulas [4]. IV cannulas can reduce the number of vascular punctures, incidences of extravasation, and the nursing workload [5]. Additionally, IV cannulas are soft and not prone to puncture the blood vessels, thus they have gained wide use in fluid infusion and treatment of the frail elderly, critically ill patients, and patients receiving intravenous nutritional support [6].

However, the failure rate of IV cannula placement is as high as $69 \%$ [7]. Reasons for the failure include accidental removal or displacement, pain, phlebitis, occlusion, infiltration, and infection $[8,9,10]$. Removal of IV cannulas before completing venous therapy places a higher cost burden on the patient and makes the patient subject to discomfort and pain due to re-puncture. To reduce the occurrence of medical disputes and to ensure the safety of patients, the two departments of the department of extra renal thoracic surgery and hepatobiliary surgery of our hospital adopted the quality control circle activity guided by the Deming circulation management method in the continuous quality improvement activity fornurses'use of intravenous indwelling needlesfrom March 1, 2019, and December 30, 2019.

\section{Methods}

\subsection{Study Design}

To evaluate the effectiveness of the method for improving the use of intravenous indwelling needles, we used a case-control study. Participants were 2,472 hospital patients who underwent IV cannula placement from March 2019 to June 2019.

\subsection{Participants}

The control group included 1,035 patients in the Departmentof Hepatobiliary Surgery and the Department of Renal and Thoracic Surgery of our hospital. The test group included 1,437 patients who underwent IV cannula placement in the same places as above but from July 2019 to December 2019.

\subsection{Inclusion Criteria}

Inclusion criteria were: (1) patients who underwent IV cannula infusion; (2) patients who had full communication capability and signed an informed consent form to voluntarily participate in the present study. Exclusion criteria were: (1) patients with edema and hypoproteinemia; (2) patients with psychiatric disorders; (3) patients who underwent infusion of chemotherapy drugs; (4) patients with other wounds, such as scratches and burns on the skin of the puncture site.

\subsection{Methods}

The control group was provided with routine nursing. In addition to routine nursing, the test group was subjected to the PDCA Cycle, which consisted of the following activities:

\subsubsection{Organize the Training}

Thirty-four nurses of the two departments received centralized training on intravenous indwelling needle puncture technique and maintenance skills to standardize nurse operation. Standardize the process for using a u-shaped fixed catheter, pulse punching tube, sealing tube, etc., and organize assessment after the training

\subsubsection{Improve Patient Compliance with Health Education}

The management team of venous therapy made an assessment form of IV cannula according to relevant standards and actual situation, recording details of the patient's personal information, information aboutthe IV cannula insertion, and the reason for removing the IV cannula. The questionnaire about maintenance knowledge of intravenous indwelling needle use was established, and the awareness rate of health education knowledge in patients using indwelling needle was regularly investigated. The responsible nurses once again focus on the contents unknown to the patients, and through various forms of education, improve patients' compliance with health education, enhance patients' awareness of safety, and encourage them to participate in protecting patient safety.

\subsubsection{Establish a Sound Management Mechanism}

i. The IV therapy management team developed a quality control chart of IV cannula according to relevant standards and actual conditions.

ii. The items on the chart were randomly picked for inspection by the head nurse for each month, with the IV therapy liaison nurses assessing the quality control every week, the on-duty group leader conducted quality control assessments each day, and the on-duty nurses were in charge of assessing specific items. Next, the on-duty group leader was tasked with summarizing the daily issues encountered in IV cannula placement, as well as discussing and analyzing those issues at a QCC seminar held every two weeks. The QCC team formulated corresponding improvement measures after the QCC discussion and decided to include the issues in the next PDCA cycle for resolution.

\subsection{Observation Indicators}

\subsubsection{Tangible Outcomes}

Between-group comparisons were performed on the duration of IV cannula placement, rate of complications (extravasation, phlebitis, cannula obstruction, and blood oozing), and patient awareness rates regarding maintenance knowledge for the IV cannula. We also compared patient satisfaction with the nursing staff and nurse skills in IV cannula infusion and maintenance between the study groups.

\subsubsection{Intangible Outcomes}

Intangible outcomes consisted of six aspects: QCC technique application, teamwork, communication and coordination, sense of responsibility and honor, motivation, and problem-solving ability. Each QCC member performed a self-assessment before and after the activity, and each item 
was scored 5,3 , or 1 , with a higher score indicating a stronger working ability.

\subsection{Statistical Analysis}

Data analysis and p-value calculations were performed in IBM SPSS 24.0 software. The survey results were analyzed using descriptive statistical methods. Count data were expressed as percentages (\%) and subjected to $\chi^{2}$-test. Measurement data were expressed as mean \pm standard deviation and subjected to t-test, with $\mathrm{p}<0.05$ indicating a statistical difference.

\section{Results}

\subsection{Participants}

The control consisted of 484 males and 551 females, with a mean age of $38.81 \pm 19.21$ years; the test group consisted of 725 males and 712 females, with a mean age of $37.44 \pm 17.17$ years. There was no statistical significance $(p>0.05)$ between the two groups in the distribution of gender and age.

\subsection{Tangible Outcomes}

\subsubsection{Between-Group Comparison of Duration of IV Cannula Placement}

The test group had a duration of $70.22 \pm 48.33 \mathrm{~h}$ for IV cannula placement, which was significantly longer $(p<0.05)$ than the duration of $60.59 \pm 41.9 \mathrm{~h}$ in the control group, as shown in Table 1

Table 1. Between-group comparison of duration of IV cannula placement.

\begin{tabular}{lll}
\hline Group & Number of subjects & Placement duration (h) \\
\hline Control group & 1,035 & $60.59 \pm 41.90$ \\
Test group & 1,437 & $70.22 \pm 48.33$ \\
p-value & $/$ & $\mathrm{p}<0.05$ \\
\hline
\end{tabular}

\subsubsection{Between-Group Comparison of Complications Due to} IV Cannula Placement

The test group had a significantly lower $(\mathrm{p}<0.05)$ rate of complications $(12.9 \%, 12.6 \%$, and $1.3 \%$ for extravasation, phlebitis, and cannula obstruction, respectively) than the control group $(30.0 \%, 11.4 \%$, and $4.1 \%$ for blood oozing, phlebitis, and cannula Obstruction respectively), as shown in Table 2.

Table 2. Between-group comparison of complication rate. Values before brackets refer to the numbers of cases, and values in brackets refer to the percentage as $n(\%)$.

\begin{tabular}{|c|c|c|c|c|c|}
\hline Group & Number of subjects & Extra-vasation & Phlebitis & Cannula obstruction & Blood oozing \\
\hline Control group & 1,035 & $311(30.0)$ & $118(11.4)$ & $42(4.1)$ & $25(2.4)$ \\
\hline Test group & 1,437 & $185(12.9)$ & $181(12.6)$ & $18(1.3)$ & $19(1.3)$ \\
\hline$\chi^{2}$-value & & 110.645 & 19.99 & 44.113 & 0.808 \\
\hline p-value & & 0.000 & 0.008 & 0.043 & 0.369 \\
\hline
\end{tabular}

\subsubsection{Between-Group Comparison of Patient Awareness Rate of Maintenance Knowledge of IV Cannula and Patient} Satisfaction Rate with Nursing Staff

Patient awareness rate of maintenance knowledge of IV cannula and patient satisfaction rate with nursing staff were $90 \%$ and $96.3 \%$, respectively, in the test group, which were significantly higher $(\mathrm{p}<0.05)$ than the counterparts of $82.4 \%$ and $81.5 \%$ in the control group, as shown in Table 3.

Table 3. Between-group comparison of patient awareness rate of maintenance knowledge of IV cannula and patient satisfaction rate with nursing staff.

\begin{tabular}{lllll}
\hline Duration & $\begin{array}{l}\text { Number of patients } \\
\text { surveyed }\end{array}$ & $\begin{array}{l}\text { Total number of } \\
\text { items }\end{array}$ & $\begin{array}{l}\text { Number of items that the } \\
\text { patients are aware of }\end{array}$ & $\begin{array}{l}\text { Awareness rate } \\
(\%)\end{array}$ \\
\hline Control group & 54 & 540 & 445 & 82.4 \\
Test group & 54 & 540 & 486 & 81.5 \\
p-value & & & 96.3 & 90 \\
\hline
\end{tabular}

\subsubsection{Between-Group Comparison of Nurse Skills in IV Cannula Infusion and Maintenance}

Table 4. Between-group comparison of nurse skills in IV cannula infusion and maintenance.

\begin{tabular}{|c|c|c|c|c|}
\hline Item & Pre-activity $(n=34)$ & Post-activity $(\mathrm{n}=34)$ & $\mathbf{t}$ & $\mathbf{p}$ \\
\hline Skill for IV cannula infusion & $85.5 \pm 2.7$ & $90.5 \pm 1.3$ & -9.934 & $<0.01$ \\
\hline Skill for IV cannula maintenance & $81 \pm 1.0$ & $93.6 \pm 2.0$ & -33.248 & $<0.01$ \\
\hline
\end{tabular}

\subsection{Intangible Outcomes}

The average age of the 11 members of the quality control circle was (33.9 \pm 5.6$)$ years old, 10 females and 1 male. The self-assessment scores of 11 QCC members were improved after QCC activities in the five aspects of QCC: technique application, communication and coordination, sense of responsibility and honor, motivation, and problem-solving ability ( $\mathrm{p}<0.05)$, as shown in Table 5 . 
Table 5. Self-assessment scores of QCC members before and after.

\begin{tabular}{lllll}
\hline Item & pre-activity $(\mathbf{n}=\mathbf{1 1})$ & post-activity $(\mathbf{n}=\mathbf{1 1})$ & $\mathbf{t}$ & $\mathbf{P}$ \\
\hline QCC technique & $2.6 \pm 1.2$ & $3.7 \pm 1.0$ & -2.301 & $0.032^{*}$ \\
Teamwork & $2.8 \pm 1.1$ & $3.7 \pm 1.0$ & -2.041 & 0.055 \\
Communication and coordination & $3.0 \pm 0.9$ & $3.9 \pm 1.0$ & -2.193 & $0.040^{*}$ \\
Responsibility and honor & $2.8 \pm 1.1$ & $4.1 \pm 1.0$ & -2.811 & $0.011^{*}$ \\
Motivation & $2.5 \pm 0.9$ & $4.1 \pm 1.0$ & -3.873 & $0.001^{*}$ \\
Problem-solving ability & $2.6 \pm 0.8$ & $3.7 \pm 1.0$ & -2.798 & $0.011^{*}$ \\
\hline
\end{tabular}

\section{Discussion}

\subsection{Deming Circulation Can Prolong Intravenous Indwelling Time and Reduce the Incidence of Complications}

Intravenous medication is characterized by a strong effect with a fast onset, and it has become an important mode of clinical treatment. Guo et al. pointed out that IV cannula placement reduces the pain of repeated punctures, which ensures puncture safety for the patients [11]. During the implementation of quality control circle nursing, strengthening patient education and preparation before puncture can reduce patients' fear and increase their active cooperation with the puncture. These informed patients also understand the occurrence of adverse reactions and complications. Wang Fang et al. [12] showed that PDCA circulation was beneficial to prolong the indwelling time of intravenous indwelling needle. Zhong Yuqing et al. [13] showed that PDCA circulation could reduce the incidence of adverse events in patients with intravenous indwelling needles. The results in Tables 1 and 2 showed that the duration of intravenous indwelling needles was longer and the incidence of complications was lower than that in the control group. The results showed that Deming circulation could prolong the indwelling duration for intravenous indwelling needles and reduce the incidence of complications.

\subsection{Deming Circulation Improves the Compliance of Patients with Health Education}

The responsible nurse conducted a regular survey on the awareness rate of health education knowledge among the patients who used indwelling needle. The questionnaire was distributed the next morning after the catheterization to gauge the patients' health-education awareness levels; the topics that required more attention or were unknown to the patients were taughtagain. Pertinently providing ongoing propaganda and education to the patient helps focus on, and highlights, the target. Table 3 results shows that the awareness rate of maintenance of intravenous indwelling needle increased, indicating that the Deming circulation improved the compliance of patients with health education.

\subsection{Deming Cycle to Improve the Professional Level of Nursing Staff}

Based on the Deming cycle management model, the knowledge and practical operation level of nurses responsible for preventing complications related to indwelling needles was significantly improved [14-15]. The intravenous infusion puncture technique is one of the basic operations performed by nursing staff. After the implementation of Deming cycle, nursing staff's scores for using pulsed punching tubes, pulsed sealing tubes, U-shaped fixed catheters, and puncture techniques for intravenous indwelling needles improved. Table 4 shows that the scores for maintenance and operation techniques of nursing staff using intravenous indwelling needles improved, indicating that use of the Deming cycle correlates with a boost in the nursing staff's business level.

\subsection{Deming Cycle to Improve Nursing Staff Comprehensive Quality}

Deming cycle is the basic theory and method followed in total quality management, including the four stages of $\mathrm{P}$ (plan), D (execution), C (inspection), A (treatment) to achieve improvements in quality management, according to the cycle. The quality management circle activities include selecting topics, scheduling activities, evaluating situations, setting targets and goals, analyzing the current situation in relation to the goals, proposing measures and countermeasures to reach targets, implement the measures, evaluating their effectiveness, and confirming the ten steps to continuously standardize, review, and improve performance. This process requires all members to participate in problem solving, contribute to the work atmosphere, and develop the nursing staff's sense of pride responsibility, and importance.

In recent years, the Deming cycle Management and QCC activities have been gradually integrated and applied in clinical nursing practices to improve nursing outcomes. Wang Hongwei's research [16] shows that the activities of quality control circle improve the comprehensive quality of the circle members. Table 5 shows that after the activity, all the team members in this study scored according to 5, 3 and 1 in the aspects of technique application, communication and coordination, sense of responsibility and honor, enthusiasm, and problem-solving ability in the quality control circle; the scoring results were improved compared with those before the activity, indicating that the application of Deming cycle may improve the comprehensive quality of nursing staff.

\section{Conclusion}

The present study shows that QCC activities applied to IV cannula nursing under the guidance of the Deming cycle Management improved the nursing team's ability to identify, 
analyze, and solve problems, which was favorable for continuous improvement of nursing quality. In the meantime, the above practices led to a longer duration of IV cannula placement and lower rates of complications (extravasation, phlebitis, and cannula obstruction) in the test group compared with the control group, which improved the patient satisfaction rate with nursing services.

However, the improvement of nursing quality in this study was mainly aimed at patients in two departments, which did not cover the whole hospital. Therefore, the further study was on the department using intravenous indwuding needle in the whole hospital, which was the deficiency of this study.

\section{References}

[1] Li, J. (2006). Nursing Management. Beijing: People's Health Press, pp.185-187.

[2] Zhang, C. (2015). Application of quality control circle activities combined with PDCA management in infectious disease health education. Today Nurse, 22(7): 158-159.

[3] Rickard, C. M., Marsh, N., Webster J., Playford, E. G., Matthew McGrail, R., Larsen, E., et al. (2015). Securing all intravenous devices effectively in hospitalized patients the SAVE trial study protocol for a multicenter randomised controlled trial. BMJ Open, 5(9): 1-6. doi: 10.1136/bmjopen-2015-008689

[4] Zingg, W., and Pittet, D. (2009). Peripheral venous catheters: an under-evaluated problem. International Journal of Antimicrobial Agents, 34, Suppl 4: S38-42. doi 10.1016S0924-8579(09)70565-5

[5] Hu, T., Chen, D., and Wang, C. (2018). Design and application of closed thoracic drainage tube. Chinese Journal of Practical Nursing, 34 (12): 939-940.

[6] Zhang, Y. and Zhong, X. (2015). Improvement and application of the method of drainage tube fixation after thoracic and abdominal surgery. Journal of Nursing, 22 (3) 77 -78.

[7] Cooke, M., Ullman, A. J., Ray-Barruel, G., Wallis, M., Corley, A., and Rickard, C. M. (2018). Not "just: an intravenous line" Consumer perspectives on peripheral intravenous cannulation
(PIVC). An international cross-sectional survey of 25 countries.
PLoS
ONE;
13
(2): $\quad \mathrm{e} 0193436$
doi

10.1371journal.pone.0193436

[8] Rickard, C. M., McCann, D., Munnings, J., and McGrail, M. R. (2010). Routine resite of peripheral intravenous devices every 3 days did not reduce complications compared with clinically indicated resite a randomised controlled trial. BMC Medicine, 8, 53. doi 10.11861741-7015-8-53

[9] Bolton, D. (2010). Improving peripheral cannulation practice at an NHS Trust. British Journal of Nursing. 19(21):1346, 1348-50. doi 10.12968bjon.2010.19.21.79998

[10] Webster J, Clarke S, Paterson D, Hutton A, van Dyk S, Gale C, and Hopkins T. (2008) Routine care of peripheral intravenous catheters versus clinically indicated replacement randomised controlled trial. BMJ. 337(7662):a339. doi 10.1136bmj.a339. PMID 18614482; PMCID PMC2483870.

[11] Guo, J., Yang, J., Zhou, Y., and Wang, Q. (2015). Development of the short peripheral venous catheter in infusion therapy. Chinese Journal of Nursing, 50 (10): 1240-1244.

[12] Wang, F., Ye, W., and Dai, L. (2019). Effect evaluation of PDCA cycle on prolonging the indwelling time of intravenous indwelling needle. Electronic Journal of Practical Clinical Nursing, 4(4):117-118.

[13] Zhong, Y., and Liang, Y. (2020). Observation of the effectiveness of quality control circle activities in children with intravenous cannula placement under the guidance of the Deming Circle Management. Journal of Baotou Medical College, 36 (02): 92-94.

[14] Wang, W., Cui, N. (2016). Application of PDCA cycle in reducing unplanned extubation of peripheral venous indwelling needle in children. Chinese Medical Guide, 16 (7): 165-166.

[15] Zhong, X., Zhang, D., Lin, X., et al. (2017). Influence of the PDCA cycle management mode on the common complications of intravenous cannula in neonates with pneumonia and the satisfaction of the neonates' families with nursing services. Modern Diagnosis and Treatment, 28 (7): 1359-1360.

[16] Wang, H. (2020). The role of quality control circle management in improving the standard fixation rate of indwelling needle. Nursing Practice and Research, 17 (13): 144-146. 